\title{
Optimal Management Site of Hospitalization for Patients with Diabetic Ketoacidosis
}

\author{
Leonid Barski ${ }^{1 *}$, Iftach Sagy ${ }^{1}$, Yael Ben Baruch Golan ${ }^{1}$ and Yaniv Almog ${ }^{2}$ \\ ${ }^{1}$ Department of Internal Medicine F, Soroka University Medical Center, Israel \\ ${ }^{2}$ Medical Intensive Care Unit, Soroka University Medical Center, Israel
}

Submission: December 22, 2017; Published: January 09, 2018

*Corresponding author: Leonid Barski, Department of Medicine F, Soroka University Medical Center, P.O. Box 151, Beer-Sheva 84101, Israel, Tel: (972-8) 6403431; Fax: (972-8) 6400097; Email: lbarski@bgu.ac.il

\begin{abstract}
Management of patients with DKA involves rehydration, administration of insulin, correction of electrolyte derangements, correction of metabolic acidosis, and treatment of precipitant factors. To date there are no randomized prospective studies that have evaluated the optimal hospital site for the management of patients with DKA. The decision where to care for patients with DKA must be based on known clinical prognostic indicators and on the local availability of hospital resources. The response to initial therapy in the emergency department can be used as a guideline for choosing the most appropriate hospital site for further care.

The current mainstay of insulin therapy in DKA is continuous intravenous infusion. Recent studies have demonstrated the therapeutic feasibility and cost-effectiveness of the treatment of mild uncomplicated DKA with subcutaneous insulin analogs outside of the ICU setting. Optimal place of hospitalization must be based on the severity of DKA, possibility of route of administration of insulin, quality and education of medical team and medical word for appropriate monitoring and laboratory analyzes. We recommend that all patients with severe DKA, requiring intravenous insulin treatment, must be hospitalized in an ICU unit. Patients with mild to moderate DKA can be hospitalized in a medical word if it has trained staff and conditions suitable for adequate management and monitoring of these patients.
\end{abstract}

Keywords: Diabetic ketoacidosis; Diabetes mellitus; Site of care; Intensive care unit; General medical ward

Abbreviations: DKA- Diabetic Ketoacidosis; ICU: Intensive Care Unit

\section{Introduction}

In the last decade there has been significant improvement of survival among DKA patients in most developed countries with a mortality of $<1 \%$ [1-3]. However, DKA is associated with mortality rates as high as $5-9 \%$ in the elderly and in patients with severe comorbidities $[4,5]$. Though mortality from DKA is more often attributable to severe underlying illness and comorbidities [4], DKA itself is a hypercoagulable state resulting in potentially fatal complications including stroke, myocardial infarction, and disseminated intravascular coagulation [6,7]. Management involves rehydration, administration of insulin, correction of electrolyte derangements; particularly hypokalemia, correction of metabolic acidosis, and treatment of precipitant factors such as infection, pancreatitis, trauma, and myocardial infarction [8-10]. Complications of DKA management include pulmonary venous congestion and severe electrolyte imbalance. Cerebral edema represents a major potential complication, although this has been documented mostly in children.
The optimal hospital site for the management of patients with DKA (intensive care unit, general medical ward or emergency department) is an important issue. To date there are no randomized prospective studies that have evaluated this question. Given the lack of such studies, the decision where to care for patients with DKA must be based on known clinical prognostic indicators and on the local availability of hospital resources [11-13]. The response to initial therapy in the emergency department can be used as a guideline for choosing the most appropriate hospital site for further care $[10,11]$. Admission to ICU should be considered for patients with hypotension or oliguria refractory to initial rehydration and for patients with mental obtundation or coma [11]. Patients who are mildly ketotic and without severe systemic manifestations can be effectively managed in a general medical ward [11].

Different routes of insulin treatment patients with DKA and it's influence on the site of hospitalization 
Regular insulin is favored over insulin analogues. The current mainstay of insulin therapy in DKA is continuous intravenous infusion due to its rapid onset and ease of dose titration $[4,14]$. Some institutions require intravenous insulin infusions to be managed in the intensive care setting and thus some advocate for the use of subcutaneous or intramuscular injections in order to avoid an intensive care admission [4,15-17].

Insulin analogs glulisine, aspart and lispro have been reported to have equal efficacy and potency compared to regular insulin, attributable to their similar receptor binding affinity and receptor mediated clearance $[3,18,19]$, however only studies comparing the role of intravenous glulisine infusion as an alternative to intravenous infusion of regular human insulin has been performed [20]. In spite of their more rapid onset, studies comparing the pharmacokinetics and pharmacodynamics of intravenous glulisine (an ultrashort-acting insulin analogue) to intravenous regular insulin (short-acting insulin) have found thatglulisine demonstrates equivalent glucose utilisation and disposal; and a similar distribution and elimination profile to regular insulin $[3,20]$.

The attraction of subcutaneous insulin is to avoid the need for intensive care unit admission for intravenous insulin infusions and its associated high costs, although the concern is that it may not be as effective as intravenous insulin [21]. A Cochrane review in 2016 analyzed five small RCTs (total $n=201$ ) comparing subcutaneous injections of insulin analogues (lispro and aspart) to regular insulin intravenous infusions in patients with mild to moderate DKA and revealed no statistical difference in the time to resolution of DKA or rates of hypoglycemia [22].

The benefits of administering subcutaneous insulin injections hourly outside of the intensive care setting must also be weighed against the increased demands on nursing staff in medical wards, increased variability with dose administration times, time to onset of action, peak effect, and duration of effect [23].

Recent studies have demonstrated the therapeutic feasibility and cost-effectiveness of the treatment of mild uncomplicated DKA with insulin analogs outside of the ICU setting; however, the proposed subcutaneous insulin protocols have yet to find widespread support from hospital administrations and treating physicians [10]. Lack of nursing and medical staff training, presence of multiple comorbidities in diabetes patients, insufficient resources with which to conduct frequent bedside glucose testing in hospital wards, and the absence of financial incentives to treat DKA outside of ICUs are some of the factors that diminish the enthusiasm of providers to take on this important issue of health care resource utilization without compromising patient care [10]. Future trials testing less complex subcutaneous insulin delivery protocols should be considered in an attempt to simplify management of mild DKA in a non-ICU setting [10].
The use of constant intravenous insulin infusions is now generally considered to be the standard of care in most hospitals [24]. But in a large part the choice of route insulin therapy for patients with DKA is institution dependent [24].

\section{Severity of DKA and it influence on the site of hospitalization}

Diagnostic criteria for DKA include presence of blood glucose $>250 \mathrm{mg} / \mathrm{dL}$, arterial $\mathrm{pH}$ of $\leq 7.30$, bicarbonate level of $\leq 18 \mathrm{~m} \mathrm{Eq} / \mathrm{L}$, and adjusted for albumin anion gap of $>10-12$ [4]. The Joint British Diabetes Societies suggest that the presence of one or more of the following may indicate severe diabetic ketoacidosis and admission to a Level 2 / high-dependency unit environment and insertion of a central line and immediate senior review should be considered: blood ketones over $6 \mathrm{mmol} / \mathrm{l}$; bicarbonate level below $5 \mathrm{mmol} / \mathrm{l}$; venous/arterialpHbelow7.1; hypokalemia on admission (under $3.5 \mathrm{mmol} / \mathrm{l}$ ); Glasgow Coma Scale (GCS) less than 12 or abnormal AVPU (Alert, Voice, Pain, Unresponsive) scale; oxygen saturation below 92\% on air (assuming normal baseline respiratory function); systolic blood pressure below $90 \mathrm{mmHg}$; pulse over 100 or below $60 \mathrm{~b}$ min; anion gap above [25].

\section{Significance of standardized protocols for DKA management and medical staff education}

Several guidelines for the management of DKA have been suggested [4,25-27]. One approach to delivering best clinical practices is development of inpatient standardized protocols for DKA management. Studies have shown that protocol-directed care of patients with DKA is both safe and efficient, as highlighted by significant decreases in length of stay without increases in the rate of iatrogenic complications [10,28,29].

Patients treated under these protocols expe $\neg$ rienced a decrease in time to resolution of $\sim 10$ hours without increased rates of iatrogenic hypoglycemia or hypokalemia [9]. Other studies have also demonstrated suboptimal care as a result of low adherence stemming from discontinuity of medical care, understaffing, and low experience in the care of DKA patients $[30,31]$.

Therefore, there is a need for ongoing medical staff education and training in order to increase protocol adherence in patients with DKA. The care of patients with DKA should be a collaborative effort that includes the expertise of endocrinology, intensive care, medical pharmacy, and nursing specialists [10]. Recent studies showing clinical benefits and safety of subcutane $\neg$ ous insulin administration in patients with mild DKA and utility of protocol-driven care offer new pathways to reducing the cost of DKA care while maintaining quality of clinical outcomes. Also, resources should be directed toward the education of primary care providers and patients and their families so that they can identify signs and symptoms of uncontrolled diabetes earlier [3]. 


\section{Current Research in Diabetes \& Obesity Journal}

\section{Summary and Outlook}

Optimal place of hospitalization must be based on the severity of DKA, possibility of route of administration of insulin, quality and education of medical team and medical word for appropriate monitoring and laboratory analyzes.

The current available guidelines state that the most effective means of insulin delivery during DKA is a continuous infusion of regular insulin, usually referred to as continuous lowdose insulin infusion $[4,25,27]$. Therefore, all patients with severe DKA requiring intravenous insulin treatment must be hospitalized in an ICU unit. Patients with mild to moderate DKA can be hospitalized in a medical word if it has trained staff and conditions suitable for adequate management and monitoring of these patients.

The issue of cost effectiveness in the treatment of DKA is also very important as treatment with continuous intravenous insulin infusion in ICU unit is more resource intensive as compared to hourly subcutaneous insulin injection or even intravenous insulin infusion in the medical ward. Adhering to the existing protocols in the treatment of DKA including rehydration, correction of electrolyte derangements, administration of insulin, correction of metabolic acidosis, and treatment of precipitants must be performed in all institutions and wards managing patients with DKA

\section{References}

1. Chen HF, Wang CY, Lee HY, See TT, Chen MH, et al. (2010) Shortterm case fatality rate and associated factors among inpatients with diabetic ketoacidosis and hyperglycemic hyperosmolar state: a hospital-based analysis over a 15-year period. Intern Med 49(8) 729-737.

2. Umpierrez GE, Smiley D (2006) Diabetic ketoacidosis and hyperglycemia hyperosmolar syndrome. In: Clinical Diabetes. Translating Research into Practice. Fonseca VA, Ed. Saunders Elsevier, Philadelphia, PA, pp. 101-118.

3. Barski L, Kezerle L, Zeller L, Zektser M, Jotkowitz A (2013) New approaches to the use of insulin in patients with diabetic ketoacidosis. Eur J Intern Med 24(3): 213-216.

4. Kitabchi AE, Umpierrez GE, Miles JM, Fisher JN (2009) Hyperglycemic crises in adult patients with diabetes. Diabetes Care 32(7): 1335-1343.

5. Azevedo LC, Choi H, Simmonds K, Davidow J, Bagshaw SM (2014) Incidence and long-term outcomes of critically ill adult patients with moderate-to-severe diabetic ketoacidosis: retrospective matched cohort study. J Crit Care 29(6): 971-977.

6. Carr ME (2001) Diabetes mellitus: a hypercoagulable state. J Diabetes Complications 15(1): 44-54.

7. Hamblin PS, Topliss DJ, Chosich N, Lording DW, Stockigt JR (1989) Deaths associated with diabetic ketoacidosis and hyperosmolar coma. 1973-1988. Med J Aust 151(8): 439, 441-442, 444.

8. Thuzar M, Malabu UH, Tisdell B, Sangla KS (2014) Use of a standardised diabetic ketoacidosis management protocol improved clinical outcomes. Diabetes Res Clin Pract 104(1): e8-e11.

9. Hara JS, Rahbar AJ, Jeffres MN, Izuora KE (2013) Impact of a hyperglycemic crises protocol. Endocr Pract 19(6): 953-962.
10. Gosmanov AR, Gosmanova EO, Dillard Cannon E (2014) Management of adult dia-betic ketoacidosis. Diabetes Metab Syndr Obes 7: 255-264.

11. Kitabchi AE, Umpierrez GE, Murphy MB, Barrett EJ, Kreisberg RA, et al. (2001) Management of hyperglycemic crises in patients with diabetes. Diabetes Care 24(1): 131-153.

12. Henriksen OM, Prahl JB, Røder ME, Svendsen OL (2007) Treatment of diabetic ketoacidosis in adults in Denmark: a national survey. Diabetes Res Clin Pract 77(1): 113-119.

13. Barski L, Nevzorov R, Rabaev E, Jotkowitz A, Harman Boehm I, et al (2012) Diabetic ketoacidosis: clinical characteristics, precipitating factors and outcomes of care. Isr Med Assoc J 14(5): 299-303.

14. Nyenwe EA, Kitabchi AE (2016) The evolution of diabetic ketoacidosis: an update of its etiology, pathogenesis and management. Metabolism 65(4): 507-521.

15. Jervis A, Champion S, Figg G, Langley J, Adams GG (2013) Prevalence of diabetes ketoacidosis rises and still no strict treatment adherence. Curr Diabetes Rev 9(1): 54-61.

16. Burghen GA, Etteldorf JN, Fisher JN, Kitabchi AQ (1980) Comparison of high-dose and low-dose insulin by continuous intravenous infusion in the treatment of diabetic ketoacidosis in children. Diabetes Care 3(1): $15-20$.

17. Shankar V, Haque A, Churchwell KB, Russell W (2007) Insulin glargine supplementation during early management phase of diabetic ketoacidosis in children. Intensive Care Med 33(7): 1173-1178.

18. Gillies PS, Figgitt DP, Lamb HM (2000) Insulin glargine. Drugs 59(2): 253-260.

19. Hennige A, Strack V, Metzinger E, Seipke G, Häring HU, et al. (2005) Effects of new insulin analogues HMR1964 (insulin glulisine) and HMR1423 on insulin receptors. Diabetologia 48(9): 1891-1897.

20. Umpierrez GE, Jones S, Smiley D, Mulligan P, Keyler T, et al. (2009] Insulin analogs versus human insulin in the treatment of patients with diabetic ketoacidosis A randomized controlled trial. Diabetes Care 32(7): 1164-1169.

21. Tran TT, Pease A, Wood AJ, Zajac JD, Mårtensson J, et al. (2017) Review of evidence for adult diabetic ketoacidosis management protocols. Frontiers in Endocrinology 8:106.

22. Andrade Castellanos CA, Colunga Lozano LE, Delgado Figueroa N, Gonzalez Padilla DA (2016) Subcutaneous rapid-acting insulin analogues for dia-betic ketoacidosis. Cochrane Database Syst Rev (1): CD011281.

23. Umpierrez GE, Cuervo R, Karabell A, Latif K, Freire AX, et al. (2004) Treatment of diabetic ketoacidosis with subcutaneous insulin aspart. Diabetes Care 27(8): 1873-1878.

24. Haas RM, Hoffman AR (2004) Treatment of diabetic ketoacidosis: Should mode of insulin administration dictate use of intensive care facilities? Am J Med 117(5): 357-358.

25. Savage MW, Dhatariya KK, Kilvert A, Rayman G, Rees JA, et al. (2011) Joint British diabetes societies guideline for the management of diabetic ketoacidosis. Diabet Med 28(5): 508-515.

26. Kitabchi AE, Umpierrez GE, Murphy MB, Kreisberg RA (2006) Hyperglycemic crises in adult patients with diabetes: a consensus statement from the American Diabetes Association. Diabetes Care 29(12): 2739-2748.

27. Canadian Diabetes Association Clinical Practice Guidelines Expert Committee, Goguen J, Gilbert J. Hyperglycemic emergencies in adults. Can J Diabetes. 2013; 37 Suppl 1: S72-S76.

28. Bull SV, Douglas IS, Foster M, Albert RK (2007) Mandatory protocol for treating adult patients with diabetic ketoacidosis decreases intensive 


\section{Current Research in Diabetes \& Obesity Journal}

care unit and hospital lengths of stay: results of a nonrandomized trial. Crit Care Med 35(1): 41-46.

29. Waller SL, Delaney S, Strachan MW (2007) Does an integrated care pathway enhance the management of diabetic ketoacidosis? Diabet Med 24(4): 359-363

30. Solá E, Garzón S, García Torres S, Cubells P, Morillas C, et al. (2006)
Management of diabetic ketoacidosis in a teaching hospital. Acta Diabetol 43(4): 127-130.

31. Singh RK, Perros P, Frier BM (1997) Hospital management of diabetic ketoacidosis: are clinical guidelines implemented effectively? Diabet Med 14(6): 482-486.

Your next submission with Juniper Publishers will reach you the below assets

- Quality Editorial service

- Swift Peer Review

- Reprints availability

- E-prints Service

- Manuscript Podcast for convenient understanding

- Global attainment for your research

- Manuscript accessibility in different formats

( Pdf, E-pub, Full Text, Audio)

- Unceasing customer service

Track the below URL for one-step submission https://juniperpublishers.com/online-submission.php 